\title{
Editorial
}

Z. Epileptol. $2020 \cdot 33: 247-248$

https://doi.org/10.1007/s10309-020-00365-1

(c) Springer Medizin Verlag GmbH, ein Teil von Springer Nature 2020

Christian G. Bien ${ }^{1,2}$

'Epilepsie-Zentrum Bethel, Krankenhaus Mara, Bielefeld, Deutschland

${ }^{2}$ Labor Krone, Bad Salzuflen, Deutschland

\section{Anfälle infolge Autoimmunenzephalitiden und autoimmun-assoziierte Epilepsien}

\begin{abstract}
Noch im Jahr 2006 formulierte die Leitlinie eines europäischen Chapters der Internationalen Liga gegen Epilepsie (ILAE) zum Thema des Managements eines ersten Anfalls bei Erwachsenen: „Wenn kein Fieber besteht, ist eine Liquoruntersuchung generell kontraindiziert." [1] Diese apodiktische Position dürfte heute nicht mehr gelten. Die Entdeckung der Autoantikörper gegen neurale Oberflächenantigene bei Patienten mit Autoimmunenzephalitiden hat auch in der Epileptologie zur Erkennung von Erkrankungen geführt, die bis dahin unverstanden geblieben waren. Daher werden, anders als in der zitierten Leitlinie, Patienten mit Anfällen durchaus in unseren Kliniken punktiert.
\end{abstract}

Dabei tauchen neue Fragen auf - und genau diese sollen im vorliegenden Heft der Zeitschrift für Epileptologie behandelt werden; dabei war es mir wichtig, durchaus auch „subjektive“ Einschätzungen von Experten für Fragen zu erhalten, $\mathrm{zu}$ denen die Literatur (noch) keine "abschließende" Stellungnahme erlaubt.

Haben alle Patienten mit in irgendeiner Weise autoimmun verursachten Anfällen Epilepsie? Eine Task Force der ILAE hat soeben vorgeschlagen, zwischen akut-symptomatischen Anfällen infolge von Autoimmunenzephalitiden und autoimmun-assoziierten Epilepsien zu unterscheiden [2], was ich in einem weiteren Beitrag in diesem Heft näher darstelle [3].
Welche Patienten soll man liquorpunktieren und auf neurale Antikörper testen? Die Antwort fällt differenziert aus. Bei ersten Anfällen im Kontext subakuter Verschlechterungen des psychischen oder kognitiven Befindens sollte man beides tun; man hat dann eine relativ hohe Trefferwahrscheinlichkeit. Bei Anfallsleiden, die schon über 1 Jahr bestehen, sollte man unter besonderen Umständen auf neurale Antikörper testen, wobei dann meistens eine Serumuntersuchung ausreicht [4].

Welche pathophysiologischen Prozesse stecken hinter Autoimmunenzephalitiden? Die „mechanisms of action" der Antikörper sind sehr verschieden: Während Antikörper gegen den N-Methyl-D-Aspartat-Rezeptor (NMDAR-Ak) die erkannten Rezeptoren von der Nervenzelloberfläche verschwinden lassen, reduzieren Antikörper gegen Leucine-rich-Glioma-Inactivated-Protein 1 (LGI1) über mehrere Zwischenschritte postsynaptische a-Amino-3-Hydroxy-5-Methyl-4Isoxazolpropionsäure(AMPA-)Rezeptoren [5].

Die klinische Präsentation der in der Epileptologie häufigsten Antikörper gegen LGI1, den NMDAR und das Enzym Glutamatdekarboxylase (GAD) beschreiben 3 eigene Beiträge [6-8].

Als weitere klinische Konstellationen werden die rätselhafte Rasmussen-Enzephalitis, bei der zytotoxische T-Zellen allmählich eine der beiden Großhirnhälften zerstören, [9] und der autoimmune Status epilepticus abgehandelt [10]. 
An diagnostischen Verfahren werden besprochen: die Magnetresonanztomographie [11], die Neuropsychologie bei der limbischen Enzephalitis [12], das EEG [14] und neue Biomarker [13].

Abschließend wird das Thema diskutiert, wann Patienten mit Anfällen aufgrund autoimmuner Prozesse ihre Fahreignung zurückgewinnen können. Es werden neuartige Lösungen für dieses bisher unbearbeitete Problem vorgeschlagen [15].

Ich hoffe und wünsche, dass dieses breite Spektrum von engagiert geschriebenen Arbeiten zu einem der aktuellen Themen der Epileptologie die Leser der Zeitschrift anregt und ihrem Management betroffener Patienten neue Impulse verleiht.

\section{Korrespondenzadresse}

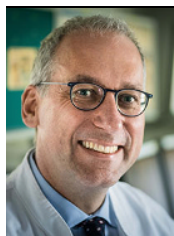

Prof. Dr. Christian G. Bien

Epilepsie-Zentrum Bethel,

Krankenhaus Mara

Maraweg 17-21,

33617 Bielefeld, Deutschland christian.bien@gmx.de

Interessenkonflikt. C.G. Bien erhielt Honorare für Fortbildungsvorträge von UCB (Monheim) und Desitin (Hamburg). Er erhält Forschungsunterstützung von der Deutschen Forschungsgemeinschaft (Bonn - BI1254/8-1, BI1254/9-1) und der Gerd-AltenhofStiftung im Deutschen Stiftungszentrum (Essen T0465/28102/2016/sm).

\section{Literatur}

1. Beghi E, De Maria G, Gobbi G, Veneselli E (2006) Diagnosis and treatment of the first epileptic seizure: guidelines of the Italian League against Epilepsy. Epilepsia 47(Suppl 5):2-8

2. Steriade C, Britton J, Dale RC, Gadoth A, Irani SR, Linnoila J, McKeon A, Shao XQ, Venegas V, Bien CG (2020) Acute symptomatic seizures secondary to autoimmune encephalitis and autoimmuneassociated epilepsy: conceptual definitions. Epilepsia. https://doi.org/10.1111/epi.16571

3. Bien CG (2020) Akut-symptomatische Anfälle infolge von Autoimmunenzephalitiden und autoimmun-assoziierte Epilepsien: Konzeptionelle Definitionen.ZEpileptol. https://doi.org/10.1007/ s10309-020-00362-4

4. Bien CG (2020) Liquor- und Antikörperdiagnostik bei Verdacht auf eine autoimmune Ursache von Anfällen. Z Epileptol. https://doi.org/10.1007/ s10309-020-00352-6

5. Chung H-Y, Wickel J, Geis C (2020) Autoimmun induzierte Anfälle und Epilepsien: ein pathophysiologisch begründetes Konzept. Z Epileptol. https://doi.org/10.1007/s10309-020-00364-2
6. JochmannE, Kunze A(2020) Anti-LGl1-Enzephalitis in der Epileptologie. Z Epileptol. https://doi.org/ 10.1007/s10309-020-00357-1

7. Räuber S, Melzer N (2020) Anti-NMDAR-Enzephalitis in der Epileptologie.ZEpileptol. https://doi.org/ 10.1007/s10309-020-00361-5

8. Malter M (2020) Epilepsie mit Antikörpern gegen Glutamat-Decarboxylase (GAD). Z Epileptol. https://doi.org/10.1007/s10309-020-00363-3

9. Cloppenborg T, Fauser S (2020) Rasmussen-Enzephalitis: praktische Aspekte zur Diagnosestellung und Therapie.ZEpileptol.https://doi.org/10.1007/ s10309-020-00359-z

10. Langenbruch L, Kovac S (2020) Autoimmuner Status epilepticus. Z Epileptol. https://doi.org/10. 1007/s10309-020-00358-0

11. Woermann F (2020) MRT bei limbischer Enzephalitis - spezifische Befunde bei einer klinisch relativ eindeutigen Form einer autoimmunen Enzephalitis? ZEpileptol. https://doi.org/10.1007/ s10309-020-00353-5

12. Helmstaedter C, Witt J-A (2020) Neuropsychologische Diagnostik bei limbischer Encephalitis. Z Epileptol. https://doi.org/10.1007/s10309-020 00354-4

13. Hansen N, Timäus C (2020) Neues zu biologischen Markern bei Autoimmunenzephalitis. Z Epileptol. https://doi.org/10.1007/s10309-020-00356-2

14. Rüegg S (2020) EEG bei Autoimmunencephalitiden. Z Epileptol. https://doi.org/10.1007/ s10309-020-00355-3

15. Specht U, Bien CG (2020) Fahreignung bei autoimmunen Anfällen und autoimmun-assoziierten Epilepsien. Z Epileptol. https://doi.org/10.1007/ s10309-020-00360-6
Stigmatisierung psychischer Erkrankungen

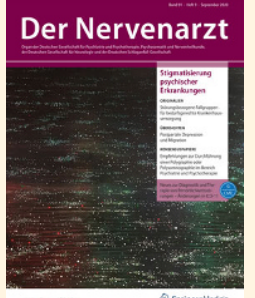

In Zeiten, in denen die Covid-19Pandemie unser gesellschaftliches Miteinander auf vielen Ebenen einschneidend verändert, wird deutlich, wie stark

dies auch unseren Umgang mit psychischer Erkrankung prägt. Das Stigma psychischer Krankheit ist nicht in erster Linie ein psychiatrisches Problem, sondern ein gesellschaftliches.

In Der Nervenarzt 09/2020 widmen sich vier Arbeiten verschiedenen Aspekten der Stigmatisierung von Menschen mit psychischen Erkrankungen und zeigen sowohl neue Richtungen wie auch praktische Implikationen der Stigmaforschung auf. Gleichzeitig belegen die Beiträge dieses Themenschwerpunkts, dass die Aufgabe noch nicht gelöst ist, und dass auch die Psychiatrie in Wissenschaft und Praxis hier ihren Beitrag leisten kann.

- Suizidstigma und Suizidprävention

- Soziale Milieus

- Qualitätsgesicherte Psychiatrie und Entstigmatisierung

- Wirkung medizinischer und psychotherapeutischer Behandlungskonzepte

Suchen Sie noch mehr zum Thema?

Mit e.Med - den maßgeschneiderten Fortbildungsabos von Springer Medizin - haben Sie Zugriff auf alle Inhalte von SpringerMedizin.de. Sie können schnell und komfortabel in den für Sie relevanten Zeitschriften recherchieren und auf alle Inhalte im Volltext zugreifen.

Weitere Infos zu e.Med finden Sie auf springermedizin.de unter „Abos" 